\title{
Research of Hydrogen Concentration Measurement in Containment Vessel of Nuclear Power Plant
}

\author{
Jiang Fu, Shengzhi Liu, Weiwei Pan, Xiqing Hu \\ System Engineering Department, Suzhou Nuclear Power Research Institute \\ Suzhou, China \\ fujiang2@cgnpc.com.cn, liushengzhi@cgnpc.com.cn, panweiwei@cgnpc.com.cn, huxiqing@cgnpc.com.cn
}

\begin{abstract}
The integrating sphere gas chamber is selected as the core of the detection system, and a new algorithm is used to calculate hydrogen gas concentration. LED light source is selected as the detection system light source, and LED s ource wavelength is processed by fiber grating and pie zoelectric ceramic, then the light source corresponding to the hydrogen gas wavelength absorption peak is obtained. The light source passes through two integrating sphere chambers of the same state at the same time, because the light inte nsity of the light sour ce is absorbed by hydrogen gas and the inner wall of the integrating sphere gas chamber, calculates the source signal by using an algorithm, and the real-time concentration of hydrogen gas obtained. Experiments show that the theoretical a lgorithm agrees well with the experimental results.
\end{abstract}

Keywords-Hydrogen; Nuclear power plant; Optical fiber sensor; Integrating Sphere; containment; Fiber grating

\section{INTRODUCTION}

China is currently the world's largest nuclear power plant in the country, but also has many operating nuclear power plants. The proportion of nuclear energy in China's energy consumption continues to rise, and nuclear energy is different from other natural energy sources. Once it is destroyed, it will cause huge irreversible losses. So the safety of nuclear power plants has always been focused. The core part of a nuclear power plant is the reactor in containment. The safe operation of the reactor is the key to ensure the normal operation of the nuclear power plant. When nuclear power plant reactor water loss accident happened, hydrogen concentration requires continuous monitoring. The probability of hydrogen overflow during the operation of the reactor is relatively high, so it is essential to strengthen the monitoring of hydrogen concentration in the surrounding environment of the reactor. If the concentration of hydrogen in the containment is not known in time, when the concentration of the overflowing hydrogen is sufficiently high, a serious safety accident such as hydrogen explosion may occur, and it will bring unimaginable damage to the environment and economy ${ }^{[1][2]}$.

At present, most researches on the detection of hydrogen volume fraction have complicated detection systems, high material costs, and extremely high demands on process manufacturing. The foreign methods of measuring the hydrogen concentration by using a fiber Bragg grating combined with a palladium membrane to detect hydrogen concentration has the problems of being easily affected by temperature, insufficient detection accuracy, and insufficient stability and repeatability ${ }^{[3][4]}$. In this paper, for the detection of hydrogen concentration, the original integrating sphere is selected as the core component of the detection system, in a natural environment, the hydrogen concentration diffused into the integrating sphere can be detected, overcome the disadvantage that the traditional method is easy to be affected by temperature, the structure is simple, and in addition, the use of LED light source is combined, and the cost is low, so that the accuracy of hydrogen gas detection results meets the requirements, and the stability and repeatability are good.

\section{HYDROGEN VOLUME FRACTION DETECTION SYSTEM WORKING PRINCIPLE}

\section{A. Gas Volume Fraction Detection Principle}

According to Birer-Lambert theorem ${ }^{[5]}$, most of the gas absorption peaks correspond to light sources of a certain wavelength. When the light source passes through the gas, the gas will absorb it, causing a certain degree of attenuation of the light intensity. If it is specified that the input light intensity corresponding to the gas absorption line is $\varphi_{i n}$ and the 
output light intensity is $\varphi_{\text {out }}$, then there is a functional relationship between the gas concentration $\mathrm{C}$ and the input light intensity and the output light intensity, as follows:

$$
\varphi_{\text {out }}=\varphi_{\text {in }} \exp (-\alpha \mathrm{LC})
$$

Formula: $\alpha$-- Gas Molecule Absorption Coefficient;

C-- The volume fraction of gas;

L-- Light source through gas and the length of the gas's effect.

Simplify Formula (1) to get:

$$
\mathrm{C}=-\frac{1}{\alpha \mathrm{L}} \operatorname{Ln}\left(\frac{\varphi_{\text {out }}}{\varphi_{\text {in }}}\right)
$$

When you want to calculate the gas volume fraction $\mathrm{C}$ to be measured, As long as the values of parameters $\alpha, \mathrm{L}$ 、

$\varphi_{\text {in }}$ and $\varphi_{\text {out }}$ in equation (2) are obtained, the purpose can be achieved, calculate the gas volume fraction to be measured C. By observing equation (2), the gas molar absorption coefficient is a constant value. Corresponding to the selected gas, the input light intensity $\varphi_{\text {in }}$ and the output light intensity $\varphi_{\text {out }}$ are the detection values. So if you want to improve the accuracy of the gas volume fraction, you can only achieve the goal by increasing the value of the action length $\mathrm{L}$, but at the same time, you should also consider simplifying the volume to meet the needs of the actual project.

\section{B.Light source wavelength modulation principle}

In nature, light sources of different wavelengths correspond to spectral absorption bands of different types of gas molecules, and often a spectrum absorption band of gas molecules corresponds to light sources of multiple wavelengths. Hydrogen gas is a relatively simple structure in nature, and its corresponding spectral absorption peaks are numerous. In this system, the $2469.5 \mathrm{~cm}^{-1}$ wavelength light source is selected as the incident light source because the $2469.5 \mathrm{~cm}^{-1}$ wavelength light source is closest to the near infrared region, so that the attenuation loss of the light source during the transmission of the optical fiber can be minimized. The LED light source has a wide spectral coverage, is easy to tune, and has a stable light source. By combining the fiber grating and the piezoelectric ceramic as a light source modulation mechanism, the LED light source is modulated to obtain a light source with a wavelength required by the system, which satisfies the needs of the gas volume fraction detection.

A fiber grating is a kind of modulated special fiber. It is obtained by periodically modulating the refractive index of the core region of the fiber, as if a light source filter was implanted in the fiber. After passing the light source, a certain wavelength of light can be obtained ${ }^{[6]}$. However, when the light sources of the other wavelengths pass through the fiber grating, they are strongly reflected, that is, the light transmittance is as small as the wavelength that satisfies the Bragg condition. Piezoelectric ceramic is a functional material that can convert mechanical energy and electrical energy to each other. It is sensitive and its deformation is one ten-millionth of its own volume. By applying an electric field across the piezoceramics to modulate the Bragg wavelength, affixing an FBG to a piezoceramic (PZT), the applied electric field will deform the piezoceramics, and the FBG will also expand and contract synchronously. Also changed the Bragg wavelength $^{[7][8]}$. The Bragg wavelength $\lambda_{B}$ can be expressed by Equation (3):

$$
\lambda_{B}=\lambda_{0}+R \cdot \cos \omega t
$$

In the formula:

$\lambda_{0}$-- The center wavelength corresponding to the gas absorption peak

$\omega$-- Piezoelectric ceramic modulation frequency

R-- Light source modulation amplitude

t-- Grating period

The axial force of a quartz fiber usually causes a relative change in the Bragg wavelength $\lambda_{B}$, as shown in equation (4):

$$
\Delta \lambda_{B} / \lambda_{B}=\left(1-P_{c}\right) \varepsilon=0.78
$$

In the formula:

$\Delta \lambda_{B}$-- Center wavelength shift 


\section{$\varepsilon$-- Fiber Bragg Grating Axial Strain}

$P_{c}$-- Optical fiber core effective photoelastic coefficient

The absorption coefficient of different gases will vary due to the wavelength of light. By observing the formula (4), the performance parameters such as the piezoelectric coefficient, the total elongation and the voltage range applied to the PZT are adjusted, and the Bragg wavelength suitable for the gas volume fraction detection is determined ${ }^{[9][10]}$.

The light source of any wavelength in the optical transmission spectrum utilizes the wavelength selectivity of the fiber grating and the piezoelectric ceramic to output the absorption wavelength corresponding to the absorption peak of the gas to be measured. The system modulates a stable light source with a wavelength of $2469.5 \mathrm{~cm}^{-1}$ by the above method as an incident light source for the hydrogen volume fraction detection system.

\section{HYDROGEN VOLUME FRACTION DETECTION ALGORITHM}

This system uses two integrating spheres with the same state, one as the measuring air chamber and one as the reference air chamber. The reference air chamber enters a LED light source with a center wavelength of $2600 \mathrm{~cm}^{-1}$. Incident light with a center wavelength of $2600 \mathrm{~cm}^{-1}$ is not absorbed by most gases. The light intensity of the LED light source passing through the reference air chamber is also not absorbed by the hydrogen gas, and the emitted light intensity is only loaded by the disturbance of the optical path and the attenuation of the light intensity by the integrating sphere chamber ${ }^{[11]}$.

The measuring air chamber is led to an LED light source with a central wavelength of $2469.5 \mathrm{~cm}^{-1}$. This wavelength light source corresponds to the hydrogen absorption peak.After measuring the air chamber, the light source not only carries the hydrogen concentration information, but also carries the signal of the optical path disturbance and the attenuation of the light intensity of the integrating sphere chamber.

As long as the light signal through the reference air chamber and the measurement air chamber is processed, the useful signal corresponding to the hydrogen volume fraction can be extracted, the interference signal is eliminated, and the volume fraction of hydrogen is calculated ${ }^{[12]}$.

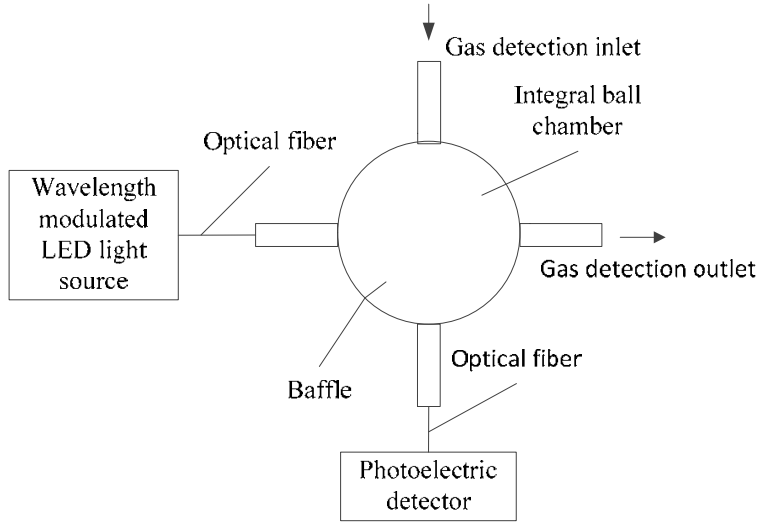

fig.1 Integrating sphere chamber

The high-reflective film material covers the surface of the integrating sphere air chamber, the light source enters the integrating sphere air chamber, and exits after multiple reflections on the inner surface of the integrating sphere. Hydrogen enters the integrating sphere air chamber from the input port, and the output port outputs. The integrating sphere is in the natural environment, there is no pressure difference in the environment, and the hydrogen is free flowing, which is more meaningful to the hydrogen detection accuracy. fig.1 shows the structure of an integrating sphere chamber.

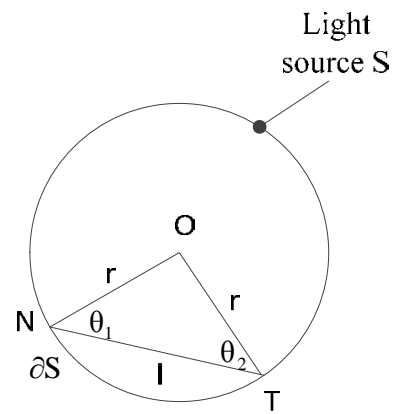

fig.2 Integrating sphere flux measurement schematic

Radiation exchange occurs between two diffuse reflection elements. Suppose that $\partial \mathrm{A}_{1}$ and $\partial A_{2}$ are two microelements on the integrating sphere, $\partial \mathrm{A}_{1}$ surface is a light emitting surface, and $\partial A_{2}$ surface is a receiving surface, then the light is emitted from the $\partial \mathrm{A}_{1}$ surface, and the light flux received on the $\partial A_{2}$ surface is: 


$$
\partial \varphi=\frac{L}{l^{2}} \cos \theta_{1} \cos \theta_{2} \partial A_{1} \partial A_{2}
$$

In formula (5),L represents the brightness of the light emitting surface.

1 represents the straight line distance between the two micro-elements $\partial \mathrm{A}_{1}$ and $\partial A_{2}$ on the ball, $\theta_{1}$ is the angle between the distance between the microelement a normal and the $\partial \mathrm{A}_{1}$ and $\partial A_{2}$ microelements, and $\theta_{2}$ is the angle between the microelement $\partial A_{2}$ normal and the distance between the $\partial \mathrm{A}_{1}$ and $\partial A_{2}$ microelements.

fig. 2 shows the schematic of the integrating sphere for detecting luminous flux. The modulated LED with a center wavelength of $2600 \mathrm{~cm}^{-1}$ is passed through an integrating sphere. The radius of the selected gas cell structure of the integrating sphere is $r$, and the diffuse reflection at each point on the inner wall of the integrating sphere is uniform. The diffuse reflection ratio of each point in the integrating sphere is $\rho$. The light source $\mathrm{S}$ is located on the structure of the integrating sphere air chamber. The total luminous flux emitted by the light source $\mathrm{S}$ is $\varphi$. $\mathrm{N}$ and $\mathrm{T}$ are two different points on the integrating sphere. Assuming that there is an area element $\partial S$ at point $\mathrm{N}$, the direct light illuminance produced on the area element is $E_{1}$. Due to the uniform diffuse reflection surface on the inner wall of the integrating sphere plenum structure, the light exit on the $\partial S$ surface is:

$$
\mathrm{M}=\rho \mathrm{E}_{1}
$$

According to Lambert's cosine law, it has the following formula:

$$
\mathrm{M}=\pi \mathrm{L}=\rho \mathrm{E}_{1}
$$

So, simplifying (7) gives the brightness of the area element:

$$
\mathrm{L}=\frac{\rho}{\pi} \mathrm{E}_{1}
$$

A diffuse reflection occurs on the $\mathrm{N}$-point area element $\partial S$. At the T-point, a secondary illuminance $\partial \mathrm{E}_{2}$ is generated, according to equation (5):

$$
\partial \mathrm{E}_{2}=\frac{\mathrm{L}}{(\mathrm{NT})^{2}} \cos ^{2} \theta \partial \mathrm{S}
$$

As shown in Fig. 2, there is a relation $\mathrm{NT}=2 r \cos \theta$ in the structure of an integrating sphere chamber,Substituting it into equation (9) is simplified:

$$
\partial \mathrm{E}_{2}=\frac{\mathrm{L}}{4 \mathrm{r}^{2} \cos ^{2} \theta} \cos ^{2} \theta \partial \mathrm{S}=\frac{\mathrm{L}}{4 \mathrm{r}^{2}} \partial \mathrm{S}
$$

According to the formula (10), the diffuse reflection of the area element $\partial S$ has a illuminance of $\frac{L}{4 r^{2}} \partial S$ at any point on the inner wall of the integrating sphere. It has nothing to do with this point in the structure of the integrating sphere air chamber. Substituting equation (8) into equation (10) is simplified as follows:

$$
\partial \mathrm{E}_{2}=\frac{\rho}{4 \pi \cdot r^{2}} \mathrm{E}_{1} \partial \mathrm{S}
$$

Except for point $\mathrm{T}$, the total illuminance generated by a diffuse reflection at each point in the gas sphere structure of the integrating sphere is:

$$
\mathrm{E}_{2}=\frac{\rho}{4 \pi \cdot r^{2}} \int_{\mathrm{S}} \mathrm{E}_{1} \partial \mathrm{S}=\frac{\rho}{4 \pi \cdot r^{2}} \varphi
$$

Assume that the entrance area of the light source on the integrating sphere air chamber structure is S1, and the area of the exit area of the light source is S2. The sphere of the entire integrating sphere is $\mathrm{S}$. When a diffuse reflection occurs, the loss occurs at the opening of the integrating gas cell structure, and the loss rate is (S1+S2)/S. Adjusting formula (12) results in:

$$
\mathrm{E}_{2}=\frac{\rho}{4 \pi \cdot r^{2}} \cdot \frac{\mathrm{S}-\mathrm{S}_{1}-\mathrm{S}_{2}}{\mathrm{~S}} \varphi
$$

Since the diffuse reflectance of each point in the integrating sphere is $\rho$, the area of the light exit and sphere of the light sphere in the integrating sphere is S2 and S, respectively, and both of them are constant values, let $\bar{\rho}=\left(1-\frac{\mathrm{S}_{1}+\mathrm{S}_{2}}{\mathrm{~S}}\right) \rho$, Get the following formula: 


$$
\mathrm{E}_{2}=\frac{\bar{\rho}}{4 \pi \cdot r^{2}} \varphi
$$

The same reasoning is:

$$
\begin{gathered}
\mathrm{E}_{3}=\mathrm{E}_{2} \frac{\rho}{4 \pi \cdot r^{2}} \int_{\mathrm{S}} \partial \mathrm{S}=\bar{\rho} \mathrm{E}_{2} \\
\mathrm{E}_{4}=\bar{\rho} \mathrm{E}_{3}=\bar{\rho}^{2} \mathrm{E}_{2}
\end{gathered}
$$

then:

$$
\mathrm{E}_{\bar{\rho}}=\frac{\bar{\rho}}{4 \pi \mathrm{r}^{2}(1-\bar{\rho})} \varphi
$$

It can be known from Equation (17) that $\mathrm{E}_{-}$is proportional to the total light flux $\varphi$ of the light source. The integrated sphere air chamber used in the system, the area of the light source entrance and the light source exit is much smaller than the spherical area, further simplified (17):

$$
\bar{\rho}=\left(1-\frac{\mathrm{S}_{1}+\mathrm{S}_{2}}{\mathrm{~S}}\right) \rho \approx \rho
$$

In the system, the reference sphere of the integrating sphere and the state of the measuring chamber are exactly the same, and the attenuation rate of the plenum structure of the integrating sphere can be obtained under the action of the incident wavelength of the central wavelength of $2600 \mathrm{~cm}^{-1}$ :

$$
\gamma=\frac{\varphi_{\text {out }}}{\varphi_{\text {in }}}=\frac{\mathrm{E}_{-} \mathrm{S}_{2}}{\varphi_{\text {in }}}=\frac{\rho}{4 \pi \mathrm{r}^{2}(1-\rho)} \mathrm{S}_{2}
$$

When hydrogen gas passes through the integrating sphere, the attenuation of the LED light source signal will increase as the hydrogen concentration increases, and the basis for further accurate calculation of the hydrogen concentration is obtained by measuring the exit light intensity and the incident light intensity.

\section{DETECTION SYSTEM COMPOSITION AND WORKING PRINCIPLE}

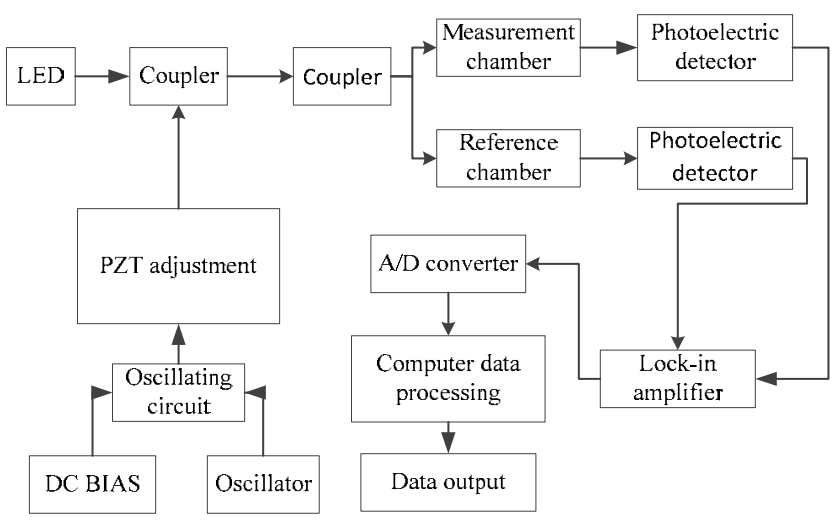

fig.3 Block diagram of hydrogen concentration detection system

As shown in fig.3 for the detection system block diagram, the first modulation of two different wavelengths of light sources to meet the needs of the system detection, One light source is a light source with a center wavelength of $2600 \mathrm{~cm}^{-1}$ as a reference light source, and the other light source is a light source with a center wavelength of $2469.5 \mathrm{~cm}^{-1}$ as a hydrogen measurement light source. The two light sources are controlled to be time-divided and alternately pass through the measurement loop in a fixed sequence.

For example, at the first time, a light source with a center wavelength of $2469.5 \mathrm{~cm}^{-1}$ is operated, When working, the directional coupler is used to split the light source into two light sources with the same status. At the same time, the air chamber and the reference air chamber are measured. Then, when the light source signal passes through the measurement chamber, in addition to the attenuation signal of the integrating sphere, the fluctuation of the optical path, and the interference signal of the measurement circuit disturbance, a signal of the hydrogen gas volume fraction is also loaded.

At the second moment, the light source with the center wavelength of $2600 \mathrm{~cm}^{-1}$ works, and when working, the directional coupler is used to split the light source into two light sources with the same status, and at the same time, the gas chamber and the reference gas chamber are measured.The reference light source emits light. Since the light source with a center wavelength of $2600 \mathrm{~cm}^{-1}$ is not absorbed by hydrogen gas, a signal of hydrogen volume fraction will not be applied after passing through the gas chamber, but an interference 
signal that attenuates the signal of the integrating sphere, fluctuates the optical path, and measures the disturbance of the loop is loaded.

By collecting the emitted light signals of different light sources through the air chamber structure of the integrating sphere, four optical signals can be obtained. This four-channel optical signal is the light source with a central wavelength of $2469.5 \mathrm{~cm}^{-1}$ and measures the amount of light emitted from the air chamber and the reference air chamber: $\varphi_{\text {out }}\left(\lambda_{1}\right)$, $\varphi_{\text {outx }}\left(\lambda_{1}\right)$. The center wavelength is $2600 \mathrm{~cm}^{-1}$. The output of the light source through the measurement chamber and the reference chamber is $\varphi_{\text {out }}\left(\lambda_{2}\right), \varphi_{\text {outx }}\left(\lambda_{2}\right)$. Where subscript $\mathrm{x}$ represents the relevant parameters of the light source through the reference air chamber; $\lambda_{1}$ represents the wavelength of light having a center wavelength of $2469.5 \mathrm{~cm}^{-1} ; \lambda_{2}$ represents the wavelength of light having a center wavelength of $2600 \mathrm{~cm}^{-1}$.

By processing these four-way signals, interference signals such as fluctuations in the signal light source, disturbances in the sensing light path, and drifts in the detection circuit can be eliminated. Extract a useful signal containing the hydrogen volume fraction.Calculated as follows:

$$
\begin{gathered}
\varphi_{\text {outx }}\left(\lambda_{2}\right)=\varphi_{\text {inx }}\left(\lambda_{2}\right) \cdot \gamma \\
\varphi_{\text {outx }}\left(\lambda_{1}\right)=\varphi_{\text {inx }}\left(\lambda_{1}\right) \cdot \gamma \\
\varphi_{\text {out }}\left(\lambda_{2}\right)=\varphi_{\text {in }}\left(\lambda_{2}\right) \cdot \gamma \\
\varphi_{\text {out }}\left(\lambda_{1}\right)=\varphi_{\text {in }}\left(\lambda_{1}\right) \cdot(\exp (-\alpha L C)+\gamma)
\end{gathered}
$$

Simplified formulas (20), (21), (22), (23), are:

$$
\frac{\varphi_{\text {outx }}\left(\lambda_{1}\right)}{\varphi_{\text {out }}\left(\lambda_{1}\right)} \cdot \frac{\varphi_{\text {out }}\left(\lambda_{2}\right)}{\varphi_{\text {out }}\left(\lambda_{2}\right)}=\frac{\gamma}{\exp (-\alpha L C)+\gamma}
$$

In many interference signals, the rate of the integrating sphere decay is an important distraction item in the detection system. The center wavelength is $2600 \mathrm{~cm}^{-1}$. The light source is mainly loaded with the integrating sphere attenuation rate signal through the gas chamber. The decay rate of the integrating sphere has been calculated theoretically in equation (19). Combining Equation (2) with the simplified equation (24), the hydrogen volume fraction $C$ value is expressed as follows:

$$
C=\frac{1}{\alpha L} \operatorname{Ln}\left(\frac{\varphi_{\text {oux }}\left(\lambda_{1}\right) \cdot \varphi_{\text {out }}\left(\lambda_{2}\right)}{\varphi_{\text {out }}\left(\lambda_{1}\right) \varphi_{\text {out }}\left(\lambda_{2}\right)-\varphi_{\text {outx }}\left(\lambda_{1}\right) \cdot \varphi_{\text {out }}\left(\lambda_{2}\right)} \cdot \frac{4 \pi r^{2}(1-\rho)}{\rho S_{2}}\right)
$$

Photodetectors in the inspection system convert light signals to voltage values for later calculation, The converted voltage value is input to the lock-in amplifier, Finally, the digital signal corresponding to the optical signal can be obtained through the A/D DAC. These digital quantities can be processed directly using a computer. The result of the processing is a real-time hydrogen volume fraction.

\section{V.EXPERIMENTAL ANALYSIS OF HYDROGEN VOLUME FRACTION}

First of all, the assembled system is vacuumed and calibrated to make the system stable at zero state. In the experiment, different volume fractions of hydrogen are injected into the gas chamber for measurement to identify the stability of the detection system. The experimental results shown in fig. 4 are a good example of the accuracy of the algorithm and the system.

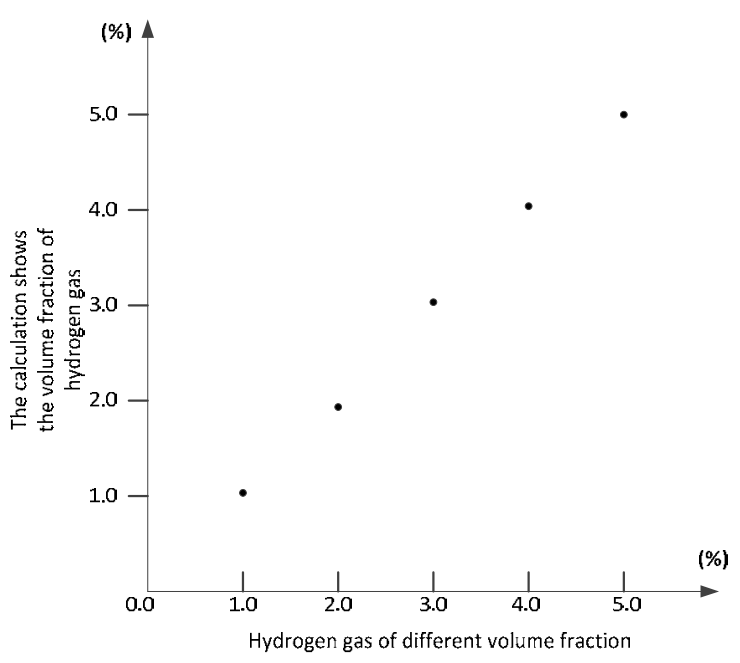

fig.4 Comparison diagram of hydrogen concentration

After the theoretical algorithm and the stability of the system are verified, it is also verified that the repeatability of the test is good enough. Restore the system to the same environment as in the zero state. The hydrogen standard gas with a volume fraction of $1.0 \%$ was injected multiple times in 
succession. After the value to be displayed was stable, the measured values $\mathrm{Ci}$ were recorded as shown in Table I.

Table I Hydrogen gas repeatability experiment data

\begin{tabular}{|c|c|c|c|c|c|c|c|c|c|c|}
\hline $\begin{array}{c}\text { The measureme } \\
\text { times }\end{array}$ & nt 1 & 2 & 3 & 4 & 5 & 6 & 7 & 8 & 9 & 10 \\
\hline $\begin{array}{l}\text { Displayed } \\
\text { value(\%) }\end{array}$ & 1.002 & 0.998 & 1.001 & 1.002 & 0.999 & 1.001 & 1.003 & 1.001 & 0.999 & 1.001 \\
\hline $\begin{array}{c}\text { Average } \\
\text { value }\end{array}$ & & & & 1.00 & & & & & & \\
\hline
\end{tabular}

The standard deviation $\sigma$ of a single measurement is obtained from the following formula:

$$
\sigma=\sqrt{\frac{\sum_{i=1}^{n=10}\left(C_{i}-\bar{C}\right)^{2}}{n-1}}
$$

In the formula: Ci-- is the ith measurement value;

$$
\bar{C} \text {--the arithmetic average of } 10 \text { consecutive }
$$

measurements;

$$
\mathrm{n} \text {-- continuous measurement times. }
$$

The calculation result based on the collected data in Table I is $\sigma=0.006$.

Relative standard deviation is:

$$
C_{v}=\frac{\sigma}{\bar{C}} \times 100 \%
$$

Calculate $C_{v}=0.6 \%$

Through the above experiments, it has been shown that the theoretical algorithm and system have good stability, high accuracy, and good repeatability. The theoretical value is in good agreement with the actual value and meets the actual engineering requirements.

\section{CONCLUSION}

The LED light source has high stability, wide spectral coverage, and easy modulation. The combination of the fiber grating and the piezoelectric ceramic to modulate the light source fully meets the needs of the system for detecting hydrogen. The integrating sphere has a simple structure, high sensitivity and stability, and is not affected by the environment. Using different wavelength light sources to alternately pass through the measurement chamber and the reference chamber, combining the excellent theoretical algorithms, the interference signals such as the fluctuation of the signal light source, the perturbation of the sensing light path, and the drift of the detection circuit are ingeniously eliminated, and useful signals of the hydrogen volume fraction are extracted. Then using the optical fiber's excellent transmission performance, long-distance input to the lock-in amplifier and photoelectric converter for processing. Finally, the hydrogen volume fraction obtained by the computer provides a new method and new approach for hydrogen detection in nuclear power plants.

\section{REFERENCES}

[1] Wu Jian, Li Qiuju. Nuclear Power Plant Class 1E Hy-drogen Monitor and Measurement System [J].Security,2000,21 (1) :1-2.

[2] Fang Likai, Chen Song, Zhou Quanfu,etal. Analysis of Hy-drogen Distribution and Control in Nuclear Power Plant Containment under Severe Accidents [J]. Nuclear Power Engineering,2006,27 (1) :1-2.

[3] LI Zhenzhen. Research on Microlens Optical Fiber Hy-drogen Sensor [D]. Wuhan University of Technology Master Thesis, Hubei: Wuhan University of Technology,2010:1-8.

[4] Zhang Yi, Zhuang Zhi, Li Qisheng,etal. Fiber Fabry-Perot Hydrogen Detection Technology[J]. Journal of Sensi-ng Technology,2010,23 (10) :1386-1389.

[5] Zhang Jingchao, Guan Lijun. Methane Concentration Detection Based on Near Infrared Absorption Principle[D].Yanshan University Master Thesis,Hebei: Yanshan University,2008:19-20.

[6] Zhang Gang, Wang Jun, Sa Jiming,etal. Spectral Absorption Based Wavelength Stability Control of Optical Fiber Gas Sensors[J]. Instrument Technology and Sensors,2008,3:4-5.

[7] Zhang Jilong, Yan Xiaomei. Research on DFB Laser Drive and Temperature Control System for Gas Detection[D]. North Central University Master Thesis,Shanxi: North Central University,2007:1-2.

[8] Wu Jing.Absorption-type gas sensor gas cell design ov-erview [J].China Hi-tech,2009,122 (11) :18-19.

[9] Zhu Ping, Liu Guangda, Tian Lei,etal. Design and App-lication of Lock-in Amplifier in Optical Fiber Gas Detec-tion [J].Instrument Technology and Sensors,2007,3:49-51.

[10] Liu Quan, Lin Haiyan. Optical Fiber Acetylene Gas Detection System [J].Sensor Technology,2003,22(4):15-17.

[11] Wang Yutian, Guo Zengjun, Wang Litian,etal. Transm-ission Optical Fiber Methane Gas Sensor[J]. Journal of Sensing Technology,2001(2):147-151.

[12] Shang Ying, Wei Yubin, Wang Chang,etal. Research on on-line detection system of acetylene gas concentration based on absorption spectrum [J]. Journal of Sensing Technology,2010,23(2):171-174. 